\title{
Potential magnetic fields around flux ropes
}

\author{
M. Vandas ${ }^{1, \star}$, E. P. Romashets ${ }^{2}$, and S. Watari ${ }^{3}$ \\ 1 Astronomical Institute, Academy of Sciences, Boční II 1401, 14131 Praha 4, Czech Republic \\ 2 Institute of Terrestrial Magnetism, Ionosphere, and Radio Wave Propagation, Russian Academy of Sciences, Troitsk, \\ Moscow Region, 142092, Russia \\ e-mail: romash@izmiran.rssi.ru \\ 3 Communications Research Laboratory, 4-2-1 Nukuikita, Koganei, Tokyo 184-8795, Japan \\ e-mail: watari@crl.go.jp
}

Received 19 September 2002 / Accepted 8 September 2003

\begin{abstract}
A method of calculation of potential magnetic fields around cylindrical and toroidal flux ropes is presented. The flux rope has an arbitrary orientation with respect to ambient field. The problem is solved analytically. Expressions for the magnetic field components, diamagnetic force, and its moment are found. Results obtained can be used for estimations of magnetic forces in systems where magnetic flux ropes and potential fields coexist, e.g., in the solar corona.
\end{abstract}

Key words. magnetic field - flux ropes - solar corona

\section{Introduction}

Direct measurements of coronal magnetic fields are very rare and scarce, so our views on coronal fields are based on theoretical considerations and various assumptions. The simplest assumption is to take the coronal fields as potential, i.e., currentfree (Altschuler \& Newkirk 1969; Hoeksema 1984), and for many years solar coronal magnetic fields have been modeled and are still modeled in this way. This approach proved to be useful due to inferred relationships between obtained magnetic field configurations and, e.g., global coronal density structures, positions of coronal holes and the heliospheric current sheet, and magnitude of the solar wind velocity (as listed by Zhao \& Hoeksema 1994). However, the absence of currents means no magnetic forces and hence no static density and temperature structures in the corona, which is in contradiction with observations. Therefore newer coronal magnetic models try to incorporate electric currents into the corona in the form of current sheets (Shatten 1971) or horizontal volume currents (Bagenal \& Gibson 1991; Zhao \& Hoeksema 1992). Other forms of currents are also possible.

Recent high resolution EUV images of the Sun reveal that the solar corona is filled by magnetic loops in all up-to-date observable scales. Many of them probably have a helical structure of magnetic field lines, i.e., they are magnetic flux ropes, as it is argued by Klimchuk et al. (2000), that due to twist

Send offprint requests to: $\mathrm{M}$. Vandas,

e-mail: vandas@ig.cas.cz

* Also guest researcher at Communications Research Laboratory, Tokyo, Japan. coronal loops have roughly uniform and circular cross sections. The most prominent and directly observable solar flux ropes are solar prominences (Vršnak et al. 1988); it is believed that solar prominences themselves are embedded in coronal flux ropes of much larger scales (Low \& Hundhausen 1995). As a result of various eruptive processes (not well understood yet), some coronal magnetic flux ropes may be ejected upward from the solar corona and propagate in the solar wind as interplanetary flux ropes (Sarris \& Krimigis 1982; Klein \& Burlaga 1982; Marubashi 1986; Shimazu \& Marubashi 2000). The most distinct interplanetary flux ropes were named magnetic clouds (Klein \& Burlaga 1982).

The origin of flux ropes in the corona is not known. They may be formed directly in the corona due to changes in their photospheric footpoints, or they may emerge from subphotospheric levels, or both processes may occur. In any case, flux ropes in the corona surrounded by pre-existing potential or close-to-potential magnetic fields bring to the system surface currents and hence also wanted (dia)magnetic forces.

In this paper we give analytical solutions of potential magnetic fields around flux ropes of cylindrical and toroidal shapes and examine effects of diamagnetic forces. The method of determination of magnetic field configuration and diamagnetic forces is similar to that used by Parker (1957) for an oblate spheromak and by Pneuman (1983) for an elliptical flux rope, but it is more general because it makes it possible to consider an arbitrary orientation of the flux rope to the ambient field.

Calculations on a toroidal flux rope are a continuation of our previous work. Romashets \& Vandas (2001) have found a non-potential (and also not force-free) solution of a 


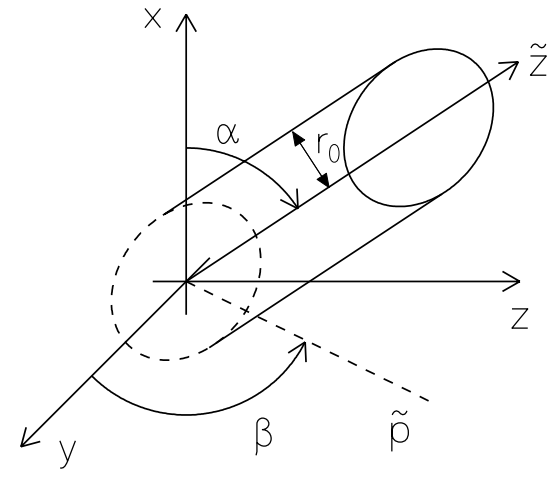

(a)

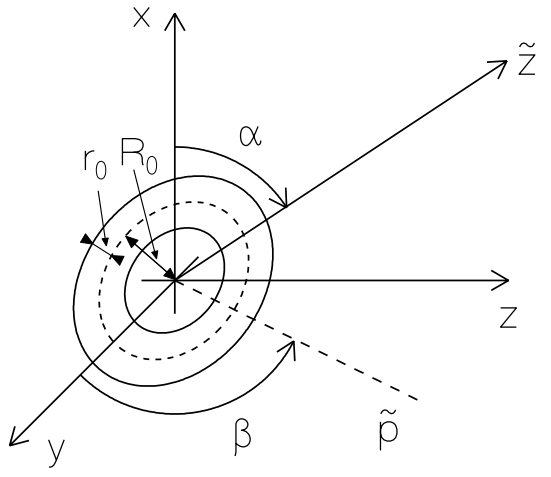

(b)

Fig. 1. Orientation of a) cylindrical and b) toroidal flux ropes in the coordinate system $x y z$ is given by two angles, $\alpha$ and $\beta$. The flux rope rotational axis is denoted by $\tilde{z}$ and its projection to the $y z$ plane by $\tilde{p}$. The cylindrical flux rope has the radius $r_{0}$, and the toroidal flux rope has the major radius $R_{0}$ and the minor radius $r_{0}$.

magnetic field configuration for a specific orientation of the toroid and the ambient field, and Romashets \& Vandas (2003) have found a potential solution for the same specific orientation (with $\alpha=90^{\circ}$ and $\beta=90^{\circ}$ according to the notation introduced in this paper in the next section).

\section{Modification of the background magnetic field by a flux rope}

We shall assume that the magnetic field around a flux rope can be described as a relatively simple potential field. This is certainly a very rough approximation of a real situation but it enables an analytical treatment. The main (homogeneous) magnetic field $\boldsymbol{B}_{\mathbf{0}}=\left(B_{0}, 0,0\right)$ is directed along the $x$ axis. A magnetic field gradient $B_{1} / L$ along the $x$ axis is added to the main field. In order to satisfy the $\operatorname{div} \boldsymbol{B}=0$ condition, there must also be a gradient in a perpendicular direction to $x$. Therefore the background magnetic field components are supposed to be

$B_{x}^{(b)}=B_{0}-\frac{B_{1}}{L} x$

$B_{y}^{(b)}=\frac{B_{1}}{L} y$,

$B_{z}^{(b)}=0$.

Parker (1957) has used such a field configuration in his analysis. A corresponding scalar magnetic potential of the field $\Phi_{0}$, $\boldsymbol{B}^{(b)}=\operatorname{grad} \Phi_{0}$, is

$\Phi_{0}=B_{0} x-\frac{B_{1}}{2 L}\left(x^{2}-y^{2}\right)$.

$\Phi_{0}$ is a harmonic function, i.e., $\Delta \Phi_{0}=0$, which ensures the condition $\operatorname{div} \boldsymbol{B}^{(b)}=0$ is fulfilled.

A cylinder or a toroid is inserted into this field with its rotational axis inclined to the main field $\boldsymbol{B}_{\mathbf{0}}$. The orientation of the rotational axis is given by two angles, $\alpha$ and $\beta$ (Fig. 1). The rotational axis makes the angle $\alpha$ with the $x$ axis, and its projection to the $y z$ plane (labeled by $\tilde{p}$ in Fig. 1) makes the angle $\beta$ with the $y$ axis; in other words, the unit vector of the rotational axis has the components $(\cos \alpha, \sin \alpha \cos \beta$, $\sin \alpha \sin \beta$ ). Denoting the axes of the intrinsic coordinate system of the cylinder or toroid as $\tilde{x}, \tilde{y}$, and $\tilde{z}$, where the $\tilde{z}$ axis is along the rotational axis and the $\tilde{y}$ axis lies in the $y z$ plane (and is perpendicular to the dashed projection line $\tilde{p}$ ), we get the following relationships between the coordinate systems:

$x=\tilde{x} \sin \alpha+\tilde{z} \cos \alpha$,

$y=-\tilde{x} \cos \alpha \cos \beta+\tilde{y} \sin \beta+\tilde{z} \sin \alpha \cos \beta$,

$z=-\tilde{x} \cos \alpha \sin \beta-\tilde{y} \cos \beta+\tilde{z} \sin \alpha \sin \beta$.

The scalar magnetic potential (4) is then expressed in the intrinsic (tilde) system through the substitutions by (5)-(7) symbolically as

$\Phi_{0}=\sum_{i=1}^{7} C_{i} \Phi_{0}^{i}$,

i.e., it is arranged as a sum of the following harmonic functions,

$\Phi_{0}^{1}=\tilde{x}, \Phi_{0}^{2}=\tilde{z}, \Phi_{0}^{3}=\tilde{x}^{2}-\tilde{y}^{2}, \Phi_{0}^{4}=\frac{\tilde{x}^{2}+\tilde{y}^{2}}{2}-\tilde{z}^{2}$,

$\Phi_{0}^{5}=\tilde{x} \tilde{y}, \Phi_{0}^{6}=\tilde{x} \tilde{z}, \Phi_{0}^{7}=\tilde{y} \tilde{z}$.

The coefficients $C_{i}$ are

$C_{1}=B_{0} \sin \alpha, C_{2}=B_{0} \cos \alpha$,

$C_{3}=\frac{B_{1}}{4 L}\left(\cos ^{2} \alpha-\sin ^{2} \alpha \cos ^{2} \beta-2 \sin ^{2} \beta\right)$,

$C_{4}=\frac{B_{1}}{2 L}\left(\cos ^{2} \alpha-\sin ^{2} \alpha \cos ^{2} \beta\right)$,

$C_{5}=-\frac{B_{1}}{L} \cos \alpha \sin \beta \cos \beta$,

$C_{6}=-\frac{B_{1}}{L} \sin \alpha \cos \alpha\left(1+\cos ^{2} \beta\right)$,

$C_{7}=\frac{B_{1}}{L} \sin \alpha \sin \beta \cos \beta$.

Now we shall look for the potential magnetic field whose normal components $B_{n}$ to the surface of the flux rope are zero (condition 1) and which approaches the undisturbed field (1)-(3) at 


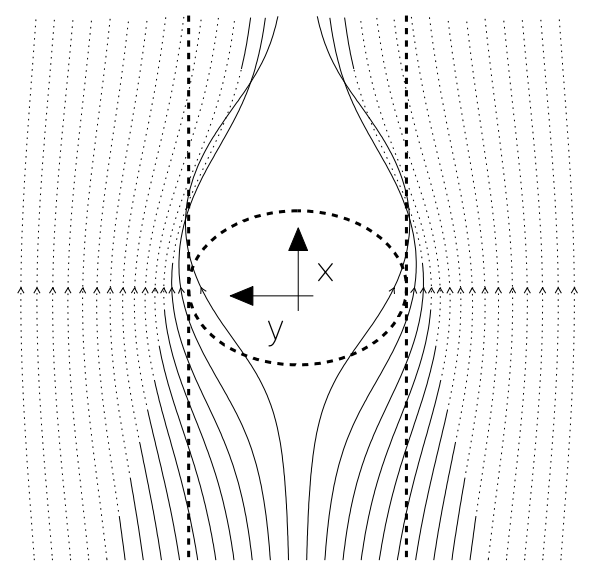

(a)

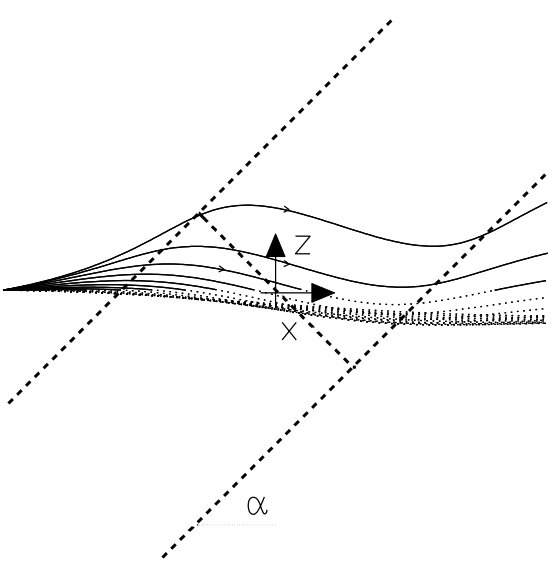

(b)

Fig. 2. Magnetic field lines around an inclined cylinder with $\alpha=45^{\circ}\left(\beta=90^{\circ}\right)$. The vertical thick dashed lines in Fig. 2a show the cylinder boundaries (extent), while the dashed ellipse is a cross section of the cylinder with the $x y$ plane. Figure $2 b$ is a side view of the field lines displayed in Fig. 2a in order to get a three dimensional imagination of the magnetic field lines behavior. The dotted lines indicate field line positions behind the plane of the Fig. 2a (i.e., with $z<0$ ).

larger distances from the rope (condition 2). That is, we add to the scalar potentials $\Phi_{0}^{i}(9)$ additional harmonic functions, for which the additional magnetic field goes to zero for large distances from the rope and for which the total magnetic field components $B_{n}^{i}=0$ at the flux rope boundary. This is done formally by changes $\Phi_{0}^{i} \rightarrow \Phi^{i}$ where $\Phi^{i}$ are harmonic functions fulfilling conditions 1 and 2 . The resulting scalar magnetic potential $\Phi$ reads

$\Phi=\sum_{i=1}^{7} C_{i} \Phi^{i}$

and the magnetic field components directly follow from

$\boldsymbol{B}=\operatorname{grad} \Phi$

Expressions for $\Phi^{i}$ and derivations of $\boldsymbol{B}$ are given separately for cylindrical and toroidal flux ropes in Appendices A and B, respectively.

\section{Magnetic field around flux ropes}

For simplicity, let us take $B_{1}=0$, that is, the ambient magnetic field is initially homogeneous. Then the model field around a cylindrical flux rope (A.10)-(A.12) reduces to simple expressions

$B_{r}=B_{0}\left(1-\frac{r_{0}^{2}}{r^{2}}\right) \cos \varphi \sin \alpha$,

$B_{\varphi}=-B_{0}\left(1+\frac{r_{0}^{2}}{r^{2}}\right) \sin \varphi \sin \alpha$,

$B_{Z}=B_{0} \cos \alpha$.

Due to symmetry the dependence on the angle $\beta$ disappears and only the dependence on the cylinder inclination $\alpha$ to the background magnetic field remains. The magnetic field lines in general are not planar; Fig. 2 shows their behavior for the case when the flux rope is inclined $45^{\circ}$ to the background field.

The maximum magnetic field increase is given from (13)-(15) by $B / B_{0}=\sqrt{1+3 \sin ^{2} \alpha}$. The angle $\alpha=90^{\circ}$ gives the largest increase of 2 . Figure 3 a shows the magnetic field lines around the cylinder for this simple case. The magnetic field lines are planar and they lie in planes parallel to the $x y$ plane. The maximum magnetic field (i.e., 2 times) is in point $M$ indicated in Fig. 3. There is also zero magnetic field strength in point $\mathrm{O}$ (null point; in fact null line in three dimensions).

Figure 3 b shows a similar picture but for a toroid. Magnetic field lines are not planar in general (planar ones are only those lying in the $x y$ plane, as is the case of Fig. 3b). One can find a similarity in the field increase along the toroid with inclined cylinders for various places at the toroid. The maximum magnetic field increase is $B / B_{0} \approx 1.9$, e.g., in point $N$ (with $z=r_{0}$ ) in Fig. $3 \mathrm{~b}$; compare it with the cylinder in point $M$ in Fig. $3 \mathrm{a}$ where $B / B_{0}=2$. This maximum value slightly depends on the aspect ratio $R_{0} / r_{0}$; the above given value was for $R_{0} / r_{0}=4$ while for $R_{0} / r_{0}=3$ it is $\approx 1.8$. In point $\mathrm{M}$ in Fig. $3 \mathrm{~b}$ the field increase is small: $B / B_{0} \approx 1.2$; compare it with the case of a cylinder parallel to $\boldsymbol{B}_{\mathbf{0}}$, i.e., having $\alpha=0^{\circ}$, where $B / B_{0}=1$. There is also zero magnetic field strength in point $O$ (null point). Figures. $4 c-4 d$ illustrate this magnetic field strength behavior. Figure 4 shows the magnetic field lines and field magnitude distribution in various $z$ planes and Fig. 5 shows the magnetic field lines for different inclinations $\alpha$.

\section{Diamagnetic force}

The general expressions (A.10)-(A.12) or (B.17)-(B.19) make it possible to calculate a diamagnetic force acting on a flux rope

$\boldsymbol{F}=-\oint \frac{B^{2}}{2 \tilde{\mu_{0}}} \boldsymbol{n} \mathrm{d} S$ 


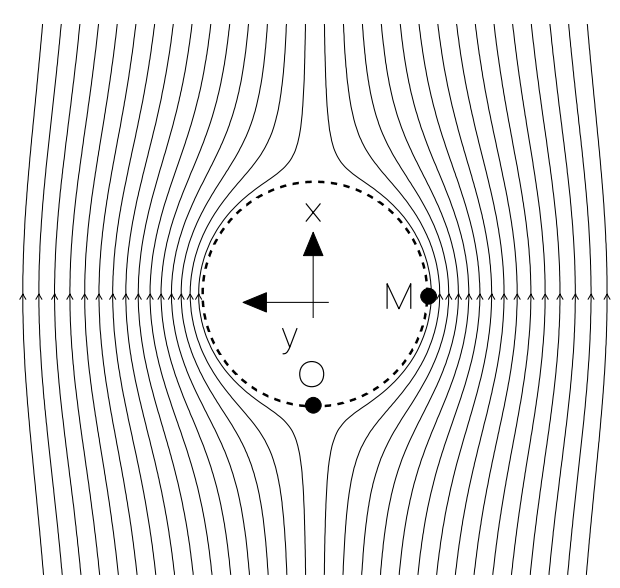

(a)

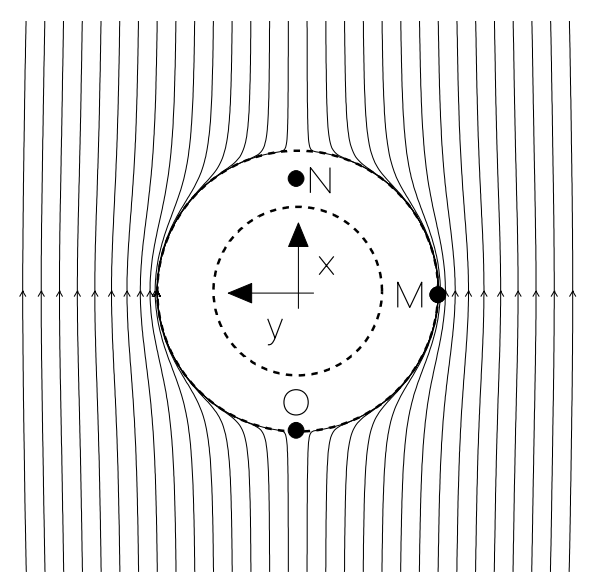

(b)

Fig. 3. Magnetic field lines in the $x y$ plane $(z=0)$ around $\mathbf{a})$ a cylinder and $\mathbf{b})$ a toroid with the axes perpendicular to the background field; the coordinate systems $x y z$ and $\tilde{x} \tilde{y} \tilde{z}$ coincide (that is, $\alpha=90^{\circ}$ and $\beta=90^{\circ}$ ). The flux ropes are drawn as thick dashed lines $\left(R_{0} / r_{0}=4, B_{1}=0\right.$ ).

where $\tilde{\mu}_{0}$ is the magnetic permeability and the integration is over the flux rope boundary. The minus sign is put because the normal unit vectors $\boldsymbol{n}$ to the flux rope surface point outward.

The diamagnetic force also acts by the moment

$\boldsymbol{M}=-\oint \frac{B^{2}}{2 \tilde{\mu_{0}}}(\boldsymbol{r} \times \boldsymbol{n}) \mathrm{d} S$,

where $\boldsymbol{r}=(x, y, z)$ is the radius vector (5)-(7). Calculations of the diamagnetic force and the moment of force are separately given for cylindrical and toroidal flux ropes in Appendices $C$ and $\mathrm{D}$, respectively.

The diamagnetic force (C.2)-(C.4) acting on a cylindrical flux rope is present only for an inhomogeneous ambient field $\left(B_{1} \neq 0\right)$ and acts in the direction $(\sin \alpha,-\cos \alpha \cos \beta$, $-\cos \alpha \sin \beta)=\boldsymbol{e}_{\tilde{x}}$, that is, in the direction of the $\tilde{x}$ axis. It lies in the $x \tilde{p}$ plane (Fig. 1) and makes an angle $90^{\circ}-\alpha$ with the $x$ axis. When the flux rope is perpendicular to the main ambient field $\boldsymbol{B}_{\mathbf{0}}\left(\alpha=90^{\circ}\right)$, then the diamagnetic force acts along this main field $\boldsymbol{B}_{\mathbf{0}}$. Such a situation is shown in Fig. 6 a.

Similarly to the diamagnetic force, the moment of diamagnetic force (C.5)-(C.7) acting on a cylindrical flux rope is also zero if there is no gradient of the ambient field, because all components of $\boldsymbol{M}$ depend on $B_{1}$ only (i.e., not on $B_{0}$ ). The moment of force is perpendicular to the flux rope axis and it is zero for the following three cases: (i) $\alpha=0^{\circ}$ or $\alpha=180^{\circ}$ (the flux rope axis is parallel to the main ambient magnetic field $\boldsymbol{B}_{\mathbf{0}}$ ); (ii) $\alpha=90^{\circ}$ and $\beta=0^{\circ}$ or $\beta=180^{\circ}$ (the flux rope axis is perpendicular to the main ambient magnetic field $\boldsymbol{B}_{\mathbf{0}}$ and it is lying in the planes of the ambient magnetic field lines given by $\boldsymbol{B}^{(b)}$ ); (iii) $\alpha=90^{\circ}$ and $\beta=90^{\circ}$ or $\beta=270^{\circ}$ (the flux rope axis is perpendicular to the main ambient magnetic field $\boldsymbol{B}_{\mathbf{0}}$ and also to the planes of the ambient magnetic field lines given by $\boldsymbol{B}^{(b)}$ ). Cases (i) and (ii) are unstable equilibrium states, the only stable equilibrium state is case (iii) (shown in Fig. 6a).

For a toroidal flux rope, the diamagnetic force (C.2)-(C.4) lies in the $x y$ plane. It is proportional to the product $B_{0} B_{1}$, therefore it is present only when a field gradient is present. For simple toroid orientations $\left(\alpha=0^{\circ}\right.$ or $90^{\circ}$, i.e., the rotational axis is parallel or perpendicular to the main ambient field $\boldsymbol{B}_{\mathbf{0}}$; see Figs. $4 \mathrm{~b}$ and $5 \mathrm{a}$ for these orientations, but note that there is no field gradient in the figures) the diamagnetic force is directed along the main ambient field $\boldsymbol{B}_{\mathbf{0}}$ (i.e., only $F_{x} \neq 0$ ).

The terms in the moment of force (D.22)-(D.24) acting on a toroidal flux rope are mostly proportional to $B_{1}^{2}$, but one term is proportional to $B_{0}^{2}$ (see $A_{\tilde{y} \eta}^{(1-2)}$ in Appendix D). Contrary to the diamagnetic force, the moment is present even if the ambient magnetic field is homogeneous. All the terms are proportional to $\sin \alpha$, therefore the moment is zero when $\alpha=0^{\circ}$ (the rotational axis is aligned to the main ambient field $\boldsymbol{B}_{\mathbf{0}}$; i.e., the orientation as in Fig. 5a). But this orientation is an unstable equilibrium state. The term with $A_{\tilde{y} \eta}^{(1-2)}$ is proportional to $\sin \alpha \cos \alpha$, so if the ambient magnetic field is homogeneous, then the moment is zero also for $\alpha=90^{\circ}$ (the rotational axis is perpendicular to the main ambient field $\boldsymbol{B}_{\mathbf{0}}$; i.e., the orientation as in Fig. 4b) and this orientation is a stable equilibrium state. When a gradient is present, the only stable equilibrium orientation is for $\alpha=90^{\circ}$ and $\beta=0^{\circ}$ or $\beta=180^{\circ}$ (the rotational axis is perpendicular to the main ambient field $\boldsymbol{B}_{\mathbf{0}}$ and aligned to the planes of ambient magnetic field lines given by $\boldsymbol{B}^{(b)}$, see Fig. 6b). Figure 7 shows the orientation of diamagnetic forces and their moments for two toroids around their stable equilibrium position (compare Fig. 7 with Fig. 6b). Because the flux rope is a loop in a real situation, this moment may cause a change of its orientation and deformations of the loop in the corona.

\section{Conclusions}

We have derived the analytical formulae of magnetic field components around cylindrical and toroidal magnetic flux ropes under the assumption that the external field is potential. Using these formulae, it is possible to calculate analytically diamagnetic forces and its moments acting on the flux ropes; we have demonstrated this and derived final expressions for these quantities. The global diamagnetic force is only present if the background field is inhomogeneous. The same is true for the 


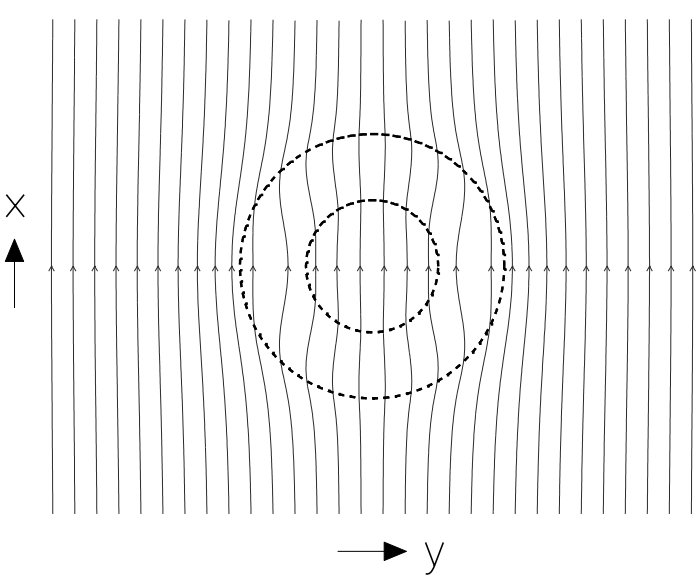

(a)

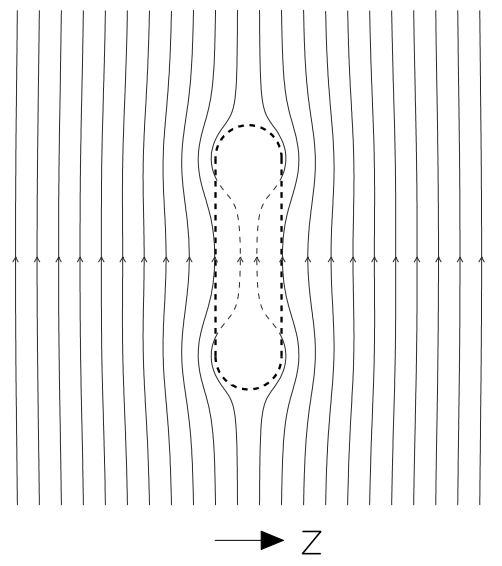

(b)

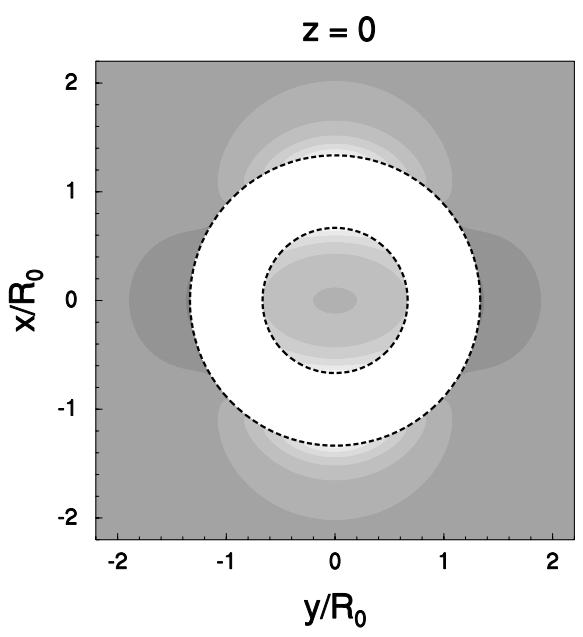

(c)

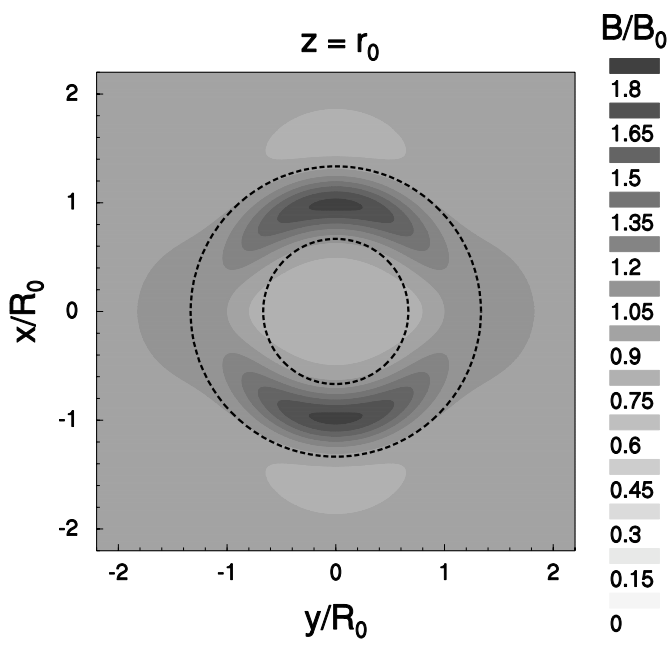

(d)

Fig. 4. Magnetic field lines and field magnitude distributions around a toroid. The toroidal flux rope is drawn as thick dashed lines. The orientation of the toroid and of the ambient field is the same as in Fig. $3 \mathrm{~b}\left(R_{0} / r_{0}=3, B_{1}=0\right)$. Figure 4a shows the magnetic field lines starting at infinity in the plane only very little shifted from $z=0$, at $z=r_{0} / 125$. Figure $4 \mathrm{~b}$ is a side view to the toroid and magnetic field lines. The parts of the magnetic field lines drawn as dashed line are located in the toroid's hole and would be hidden from our view. The toroid is in an equilibrium state because $B_{1}=0$. If $B_{1}$ was non-zero, then this orientation would be a non-equilibrium state and the moment of force would rotate the toroid by $90^{\circ}$ around the $x$ axis (the projections in a) and b) would interchange). c), d) Magnetic field magnitude distributions in two planes with different $z$.

moment of force on a cylindrical flux rope. But even a homogeneous background field acts by a global moment to a toroidal flux rope. The presented analytical solutions may be useful for estimations of magnetic field behavior around flux ropes and evaluations of magnetic forces acting on them for systems where magnetic flux ropes and potential fields coexist. For instance, such systems may probably be found in the upper solar corona. The diamagnetic force pointing upward may play a role during lift-off of coronal mass ejections (CMEs). At least some CMEs contain a flux rope (Chen et al. 1997). Another scope of application are traveling interplanetary flux ropes (magnetic clouds), which are usually approximated locally by a cylindrical or toroidal flux ropes. Our approach makes it possible to estimate magnetic forces acting on them. Analytical descriptions of a magnetic field configuration around a toroidal flux rope are useful for initialization of MHD simulations of flux rope evolution in the heliosphere. The configuration presented here was used in simulations on magnetic cloud propagation in 3D by Vandas et al. (2003).

Acknowledgements. This work was supported by grant 205/99/1712 from the Grant Agency of the Czech Republic, by grant A3003003 and project S1003006 from the Academy of Sciences of the Czech Republic, by INTAS-ESA grant 99-727, and by the Czech Republic and Japan Collaboration Research Project from the Ministry of Education, Culture, Sports, Science, and Technology of Japan.

\section{Appendix A: Magnetic field around a cylindrical flux rope}

The cylindrical system $r, \varphi$, and $Z$ of the cylinder is given in the usual way as

$\tilde{x}=r \cos \varphi, \quad \tilde{y}=r \sin \varphi, \quad \tilde{z}=Z$ 


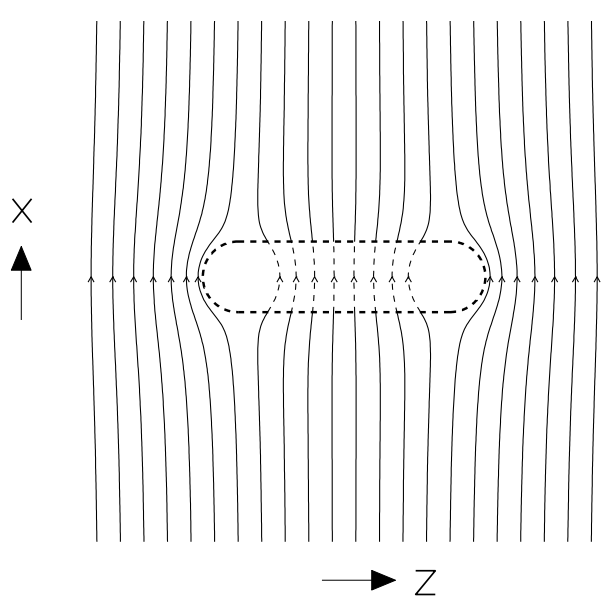

(a)

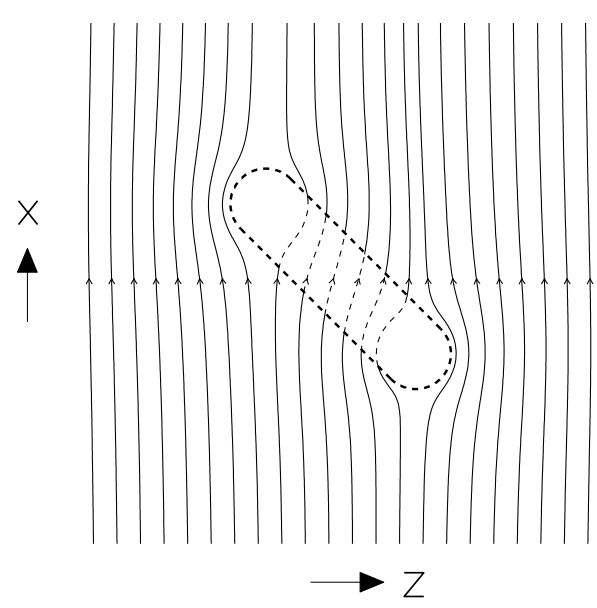

(b)

Fig. 5. Magnetic field lines around two toroids with different orientations, for a) $\alpha=0^{\circ}$, and $\left.\mathbf{b}\right) \alpha=45^{\circ}$. The flux ropes are drawn as thick dashed lines $\left(R_{0} / r_{0}=3, B_{1}=0, \beta=90^{\circ}\right)$. The parts of the magnetic field lines drawn by the dashed line are located in the toroid's hole and would be hidden from our view. The toroid in Fig. 5a is in a equilibrium state, but unstable. If perturbed, the moment of force would rotate it by $90^{\circ}$ to align it with the $x z$ plane. The toroid in Fig. $5 \mathrm{~b}$ is in a non-equilibrium state and the moment of force would also rotate it by $90^{\circ}$ to align it with the $x z$ plane. The plots supplement Fig. 4 where $\alpha=90^{\circ}$.

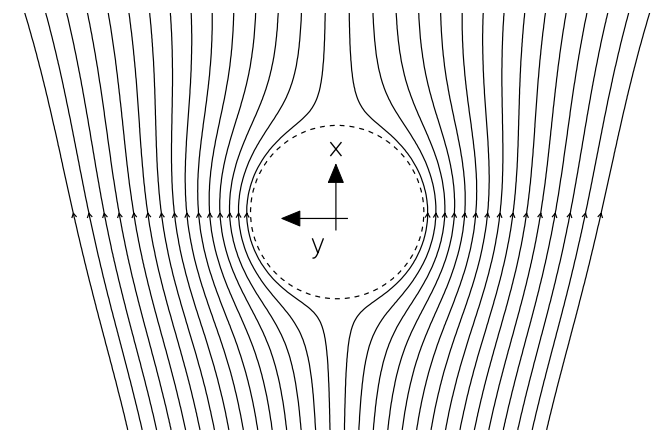

(a)

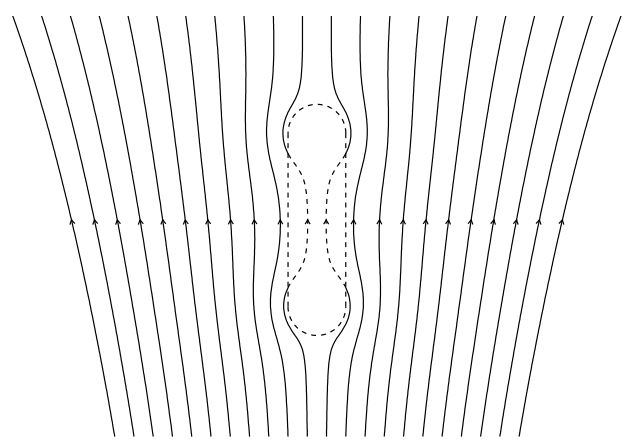

(b)

Fig. 6. a) A cylindrical flux rope surrounded by magnetic field with a small gradient $\left(B_{1} / L=1 / 12 B_{0} / r_{0}\right)$. The flux rope axis is perpendicular to the background field which is aligned to the $x y$ plane $\left(\alpha=90^{\circ}\right.$ and $\left.\beta=90^{\circ}\right)$. b) A toroidal flux rope surrounded by magnetic field with a small gradient $\left(B_{1} / L=1 / 12 B_{0} / R_{0}, R_{0} / r_{0}=3\right)$. The flux rope rotational axis is perpendicular to the main background field and aligned to the planes of the background field (parallel to the $x y$ plane; $\alpha=90^{\circ}$ and $\beta=0^{\circ}$ ). Orientations of the $x$ and $y$ axes are the same for both cases. Magnetic field lines are plotted as thin lines; the parts of the magnetic field lines marked by the dashed line are located in the toroid's hole and would be hidden from our view. The flux ropes are drawn as thick dashed lines and these cases are the only stable equilibrium positions with respect to their attitudes; a small perturbation in the axis orientation would yield a moment of force to return the flux ropes to their initial directions. The diamagnetic force points in the $x$ direction in both cases.

The harmonic functions $\Phi_{0}^{i}(9)$ are expressed in the cylindrical $\Phi^{2}=Z$, system as

$\Phi_{0}^{1}=r \cos \varphi, \Phi_{0}^{2}=Z, \Phi_{0}^{3}=r^{2} \cos 2 \varphi, \Phi_{0}^{4}=\frac{1}{2} r^{2}-Z^{2}$,

$\Phi_{0}^{5}=\frac{1}{2} r^{2} \sin 2 \varphi, \Phi_{0}^{6}=r Z \cos \varphi, \Phi_{0}^{7}=r Z \sin \varphi$.

In order to meet the conditions 1 and $2, \Phi_{0}^{i}$ are modified into $\Phi^{i}$ for which the additional magnetic field goes to zero when $r \rightarrow \infty$, and for which the total magnetic field component $B_{r}=0$ for $r=r_{0}$ :

$\Phi^{1}=r\left(1+\frac{r_{0}^{2}}{r^{2}}\right) \cos \varphi$,

$$
\begin{aligned}
& \Phi^{3}=r^{2}\left(1+\frac{r_{0}^{4}}{r^{4}}\right) \cos 2 \varphi \\
& \Phi^{4}=\frac{1}{2} r^{2}-Z^{2} \\
& \Phi^{5}=\frac{1}{2} r^{2}\left(1+\frac{r_{0}^{4}}{r^{4}}\right) \sin 2 \varphi, \\
& \Phi^{6}=r\left(1+\frac{r_{0}^{2}}{r^{2}}\right) Z \cos \varphi \\
& \Phi^{7}=r\left(1+\frac{r_{0}^{2}}{r^{2}}\right) Z \sin \varphi
\end{aligned}
$$




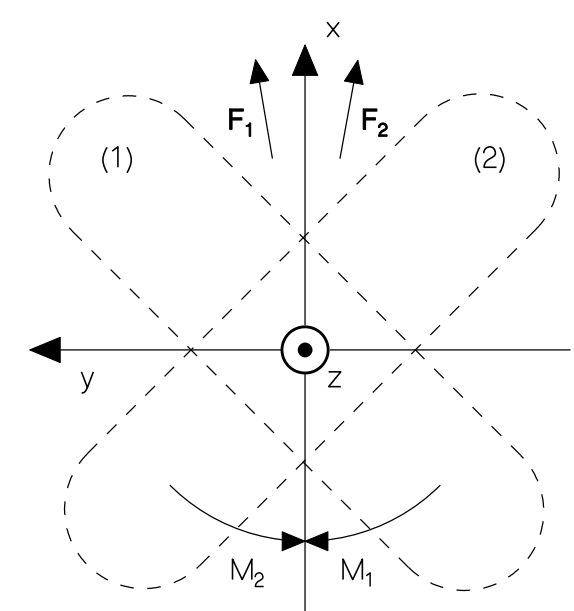

Fig. 7. Diamagnetic forces and their moments acting on toroids in an inhomogeneous background magnetic field (with $B_{1}>0$ ). Vectors of the background field are aligned to the $x y$ plane. Two toroids are plotted and labeled (1) and (2). The corresponding qualitative orientation of the diamagnetic forces are shown by the arrows and denoted by $\boldsymbol{F}_{1}$ and $\boldsymbol{F}_{2}$. Actions of the moments of forces are denoted by $M_{1}$ and $M_{2}$. They indicate that the stable equilibrium position of the toroids is at $\alpha=90^{\circ}$ and $\beta=0^{\circ}$; the toroid (1) has $\alpha_{1}=135^{\circ}, \beta_{1}=0^{\circ}$, and the toroid (2) has $\alpha_{2}=45^{\circ}, \beta_{2}=0^{\circ}$.

The magnetic field components are

$$
\begin{aligned}
& B_{r}=B_{0}\left(1-\frac{r_{0}^{2}}{r^{2}}\right) \cos \varphi \sin \alpha \\
& -\frac{B_{1}}{L}\left[\frac{1}{2} r\left(1-\frac{r_{0}^{4}}{r^{4}}\right) \cos 2 \varphi\right. \\
& \times\left(\sin ^{2} \alpha+\sin ^{2} \beta-\cos ^{2} \alpha \cos ^{2} \beta\right) \\
& +r\left(1-\frac{r_{0}^{4}}{r^{4}}\right) \sin 2 \varphi \cos \alpha \sin \beta \cos \beta \\
& -\frac{1}{2} r\left(1-\frac{r_{0}^{2}}{r^{2}}\right)\left(\cos ^{2} \alpha-\sin ^{2} \alpha \cos ^{2} \beta\right) \\
& +\left(1-\frac{r_{0}^{2}}{r^{2}}\right) Z \cos \varphi \sin \alpha \cos \alpha\left(1+\cos ^{2} \beta\right) \\
& \left.-\left(1-\frac{r_{0}^{2}}{r^{2}}\right) Z \sin \varphi \sin \alpha \sin \beta \cos \beta\right] \text {, } \\
& B_{\varphi}=-B_{0}\left(1+\frac{r_{0}^{2}}{r^{2}}\right) \sin \varphi \sin \alpha \\
& -\frac{B_{1}}{L}\left[-\frac{1}{2} r\left(1+\frac{r_{0}^{4}}{r^{4}}\right) \sin 2 \varphi\right. \\
& \times\left(\sin ^{2} \alpha+\sin ^{2} \beta-\cos ^{2} \alpha \cos ^{2} \beta\right) \\
& +r\left(1+\frac{r_{0}^{4}}{r^{4}}\right) \cos 2 \varphi \cos \alpha \sin \beta \cos \beta \\
& -\left(1+\frac{r_{0}^{2}}{r^{2}}\right) Z \sin \varphi \sin \alpha \cos \alpha\left(1+\cos ^{2} \beta\right) \\
& \left.-\left(1+\frac{r_{0}^{2}}{r^{2}}\right) Z \cos \varphi \sin \alpha \sin \beta \cos \beta\right],
\end{aligned}
$$

$$
\begin{aligned}
B_{Z}= & B_{0} \cos \alpha-\frac{B_{1}}{L}\left[Z\left(\cos ^{2} \alpha-\sin ^{2} \alpha \cos ^{2} \beta\right)\right. \\
& +r\left(1+\frac{r_{0}^{2}}{r^{2}}\right) \cos \varphi \sin \alpha \cos \alpha\left(1+\cos ^{2} \beta\right) \\
& \left.-r\left(1+\frac{r_{0}^{2}}{r^{2}}\right) \sin \varphi \sin \alpha \sin \beta \cos \beta\right] .
\end{aligned}
$$

\section{Appendix B: Magnetic field around a toroidal flux rope}

The intrinsic system of the toroid will be expressed in the toroidal coordinates $\mu, \eta$, and $\varphi$ :

$$
\begin{aligned}
& \tilde{x}=\frac{a \sinh \mu \cos \varphi}{\cosh \mu-\cos \eta} \\
& \tilde{y}=\frac{a \sinh \mu \sin \varphi}{\cosh \mu-\cos \eta} \\
& \tilde{z}=\frac{a \sin \eta}{\cosh \mu-\cos \eta}
\end{aligned}
$$

The parameter $a$ is $a=\sqrt{R_{0}^{2}-r_{0}^{2}}$, and the surface of the toroid is given by $\mu=\mu_{0}$, where $\cosh \mu_{0}=R_{0} / r_{0}$. A more detailed description of the toroidal coordinate system with an instructive figure can be found in Romashets \& Vandas (2001).

The harmonic functions $\Phi_{0}^{i}(9)$ can be expressed as a sum of toroidal harmonics

$$
\begin{aligned}
\Phi_{0}^{1}= & \sqrt{\cosh \mu-\cos \eta} \frac{2^{\frac{3}{2}} a}{\pi} \\
& \times \sum_{n=-\infty}^{\infty} Q_{n-\frac{1}{2}}^{1}(\cosh \mu) \cos n \eta \cos \varphi \\
\Phi_{0}^{2}= & \sqrt{\cosh \mu-\cos \eta} \frac{2^{\frac{3}{2}} a}{\pi} \\
& \times \sum_{n=-\infty}^{\infty} Q_{n-\frac{1}{2}}(\cosh \mu) n \sin n \eta \\
\Phi_{0}^{3}= & \sqrt{\cosh \mu-\cos \eta} \frac{2^{\frac{5}{2}} a^{2}}{3 \pi} \\
& \times \sum_{n=-\infty}^{\infty} Q_{n-\frac{1}{2}}^{2}(\cosh \mu) \cos n \eta \cos 2 \varphi \\
\Phi_{0}^{4}= & \sqrt{\cosh \mu-\cos \eta} \frac{2^{\frac{3}{2}} a^{2}}{\pi} \\
& \times \sum_{n=-\infty}^{\infty} Q_{n-\frac{1}{2}}(\cosh \mu)\left(\frac{1}{4}+n^{2}\right) \cos n \eta \\
\Phi_{0}^{6}= & \sqrt{\cosh \mu-\cos \eta} \frac{2^{\frac{5}{2}} a^{2}}{3 \pi} \\
\Phi_{0}^{5}= & \sqrt{\cosh \mu-\cos \eta} \frac{2^{\frac{3}{2}} a^{2}}{3 \pi} \\
& \times \sum_{n=-\infty}^{\infty} Q_{n-\frac{1}{2}}^{1}(\cosh \mu) n \sin n \eta \cos \varphi \\
2 & (\cosh \mu) \cos n \eta \sin 2 \varphi \\
& \\
& \\
& \\
& \\
&
\end{aligned}
$$




$$
\begin{aligned}
\Phi_{0}^{7}= & \sqrt{\cosh \mu-\cos \eta} \frac{2^{\frac{5}{2}} a^{2}}{3 \pi} \\
& \times \sum_{n=-\infty}^{\infty} Q_{n-\frac{1}{2}}^{1}(\cosh \mu) n \sin n \eta \sin \varphi
\end{aligned}
$$

$Q_{n-\frac{1}{2}}^{m}$ are the Legendre functions of the second kind. A method of how to obtain these relationships is described in Romashets \& Vandas (2001).

To meet conditions 1 and 2 , additional toroidal harmonic functions will be added to the scalar potentials $\Phi_{0}^{i}$ (B.4)-(B.10) which become negligible when $\mu \rightarrow 0$ (i.e., $\mu$ describing large distances), and for which the total magnetic field component $B_{\mu}=0$ for $\mu=\mu_{0}$. The resulting harmonics $\Phi^{i}$ are

$$
\begin{aligned}
\Phi^{1}= & \sqrt{\cosh \mu-\cos \eta} \frac{2^{\frac{3}{2}} a}{\pi} \\
& \times \sum_{n=-\infty}^{\infty}\left[Q_{n-\frac{1}{2}}^{1}(\cosh \mu)-a_{n}^{1} P_{n-\frac{1}{2}}^{1}(\cosh \mu)\right] \cos n \eta \cos \varphi
\end{aligned}
$$

and the other $\Phi^{i}$ similarly, e.g.,

$$
\begin{aligned}
\Phi^{2}= & \sqrt{\cosh \mu-\cos \eta} \frac{2^{\frac{3}{2}} a}{\pi} \\
& \times \sum_{n=-\infty}^{\infty}\left[Q_{n-\frac{1}{2}}(\cosh \mu)-a_{n}^{2} P_{n-\frac{1}{2}}(\cosh \mu)\right] n \sin n \eta .
\end{aligned}
$$

$P_{n-\frac{1}{2}}^{m}$ are the Legendre functions of the first kind. The coefficients $a_{n}^{i}$ were formally added and will be selected in order the condition $B_{\mu}=0$ for $\mu=\mu_{0}$ to be satisfied. Due to symmetry we set $a_{n}^{i}=a_{-n}^{i}$ for all $i$ and $n$.

We shall introduce the following symbols:

$\Phi^{i}=K_{i} \sqrt{\cosh \mu-\cos \eta} N_{i}(\varphi) \sum_{n=-\infty}^{\infty} G_{n}^{i}(\cosh \mu) H_{n}^{i}(\eta)$,

where

$$
\begin{aligned}
& K_{1}=\frac{2^{3 / 2} a}{\pi}, K_{2}=K_{1}, K_{3}=\frac{2^{5 / 2} a^{2}}{3 \pi}, K_{4}=\frac{2^{3 / 2} a^{2}}{\pi}, \\
& K_{5}=\frac{2^{3 / 2} a^{2}}{3 \pi}, K_{6}=K_{3}, K_{7}=K_{3}, \\
& G_{n}^{1}(x)=Q_{n-\frac{1}{2}}^{1}(x)-a_{n}^{1} P_{n-\frac{1}{2}}^{1}(x), \\
& G_{n}^{2}(x)=Q_{n-\frac{1}{2}}(x)-a_{n}^{2} P_{n-\frac{1}{2}}(x), \\
& G_{n}^{3}(x)=Q_{n-\frac{1}{2}}^{2}(x)-a_{n}^{3} P_{n-\frac{1}{2}}^{2}(x), \\
& G_{n}^{4}(x)=Q_{n-\frac{1}{2}}(x)-a_{n}^{4} P_{n-\frac{1}{2}}(x), \\
& G_{n}^{5}(x)=Q_{n-\frac{1}{2}}^{2}(x)-a_{n}^{5} P_{n-\frac{1}{2}}^{2}(x), \\
& G_{n}^{6}(x)=Q_{n-\frac{1}{2}}^{1}(x)-a_{n}^{6} P_{n-\frac{1}{2}}^{1}(x), \\
& G_{n}^{7}(x)=Q_{n-\frac{1}{2}}^{1}(x)-a_{n}^{7} P_{n-\frac{1}{2}}^{1}(x), \\
& H_{n}^{1}(\eta)=\cos n \eta, H_{n}^{2}(\eta)=n \sin n \eta, \\
& H_{n}^{3}(\eta)=H_{n}^{1}(\eta), H_{n}^{4}(\eta)=\left(n^{2}+\frac{1}{4}\right) H_{n}^{1}(\eta), \\
& H_{n}^{5}(\eta)=H_{n}^{1}(\eta), H_{n}^{6}(\eta)=H_{n}^{2}(\eta), H_{n}^{7}(\eta)=H_{n}^{2}(\eta),
\end{aligned}
$$

$$
\begin{aligned}
& N_{1}(\varphi)=\cos \varphi, N_{2}(\varphi)=1, N_{3}(\varphi)=\cos 2 \varphi \\
& N_{4}(\varphi)=N_{2}(\varphi), N_{5}(\varphi)=\sin 2 \varphi, N_{6}(\varphi)=N_{1}(\varphi), \\
& N_{7}(\varphi)=\sin \varphi
\end{aligned}
$$

It holds

$G_{n}^{i}(x)=G_{-n}^{i}(x) \quad$ and $\quad H_{n}^{i}(\eta)=H_{-n}^{i}(\eta)$.

The magnetic field components directly follow from (12):

$$
\begin{aligned}
B_{\mu}= & \frac{\sinh \mu \sqrt{\cosh \mu-\cos \eta}}{2 a} \sum_{i=1}^{7} C_{i} K_{i} N_{i}(\varphi) \sum_{n=-\infty}^{\infty}\left[G_{n}^{i}(\cosh \mu)\right. \\
& \left.+2(\cosh \mu-\cos \eta) G_{n}^{i \prime}(\cosh \mu)\right] H_{n}^{i}(\eta) \\
B_{\eta}= & \frac{\sqrt{\cosh \mu-\cos \eta}}{2 a} \sum_{i=1}^{7} C_{i} K_{i} N_{i}(\varphi) \\
& \times \sum_{n=-\infty}^{\infty} G_{n}^{i}(\cosh \mu)\left[\sin \eta H_{n}^{i}(\eta)\right. \\
& \left.+2(\cosh \mu-\cos \eta) H_{n}^{i \prime}(\eta)\right] \\
B_{\varphi}= & \frac{(\cosh \mu-\cos \eta)^{3 / 2}}{a \sinh \mu} \sum_{i=1}^{7} C_{i} K_{i} N_{i}^{\prime}(\varphi) \\
& \times \sum_{n=-\infty}^{\infty} G_{n}^{i}(\cosh \mu) H_{n}^{i}(\eta)
\end{aligned}
$$

Primes are derivatives by a function argument. Condition 2 is fulfilled by usage of the Legendre functions of the first kind (see Romashets \& Vandas 2001). Condition 1 requires to fulfill $B_{\mu}=0$ at $\mu=\mu_{0}$ for all $\eta$ and $\varphi$ in (B.17), so we must equate to zero the terms at all $\cos n \eta$ and $\sin n \eta$ in (B.17). For $i=1$ condition 1 can be symbolically expressed from (B.17) as

$\sum_{n=-\infty}^{\infty}\left[\left(R_{n}^{i}-S_{n}^{i} a_{n}^{i}\right)+2\left(T_{n}^{i}-U_{n}^{i} a_{n}^{i}\right) \cos \eta\right] \cos n \eta=0$,

where

$R_{n}^{i}=Q_{n-\frac{1}{2}}^{1}\left(\cosh \mu_{0}\right)+2 \cosh \mu_{0} Q_{n-\frac{1}{2}}^{1 \prime}\left(\cosh \mu_{0}\right)$,

$S_{n}^{i}=P_{n-\frac{1}{2}}^{1}\left(\cosh \mu_{0}\right)+2 \cosh \mu_{0} P_{n-\frac{1}{2}}^{1 \prime}\left(\cosh \mu_{0}\right)$,

$T_{n}^{i}=-Q_{n-\frac{1}{2}}^{1 \prime}\left(\cosh \mu_{0}\right)$,

$U_{n}^{i}=-P_{n-\frac{1}{2}}^{1 \prime}\left(\cosh \mu_{0}\right)$.

With the use of the relationship

$2 \cos n \eta \cos \eta=\cos (n-1) \eta+\cos (n+1) \eta$

one can express (B.20) as a sum of terms with $\cos n \eta$

$$
\begin{aligned}
\sum_{n=-\infty}^{\infty}\left(R_{n}^{i}-S_{n}^{i} a_{n}^{i}+T_{n-1}^{i}-U_{n-1}^{i} a_{n-1}^{i}+T_{n+1}^{i}\right. & \\
& \left.-U_{n+1}^{i} a_{n+1}^{i}\right) \cos n \eta=0,
\end{aligned}
$$

and equating these term to zero yields the infinite system of linear equations for $a_{n}^{i}$

$U_{n-1}^{i} a_{n-1}^{i}+S_{n}^{i} a_{n}^{i}+U_{n+1}^{i} a_{n+1}^{i}=T_{n-1}^{i}+R_{n}^{i}+T_{n+1}^{i}$. 
The coefficients $a_{n}^{i}$ are decreasing rapidly with $n$. For numerical purposes we restricted ourselves to $|n| \leq 10$ and solved a closed system (B.27) of 11 equations. The system (B.27) is the same for all $i$, only the following changes in (B.21)-(B.24) must be made:

for $i=2: Q_{n-\frac{1}{2}}^{1} \rightarrow n Q_{n-\frac{1}{2}}$ and $P_{n-\frac{1}{2}}^{1} \rightarrow n P_{n-\frac{1}{2}}$;

for $i=3: Q_{n-\frac{1}{2}}^{1} \rightarrow Q_{n-\frac{1}{2}}^{2}$ and $P_{n-\frac{1}{2}}^{1} \rightarrow P_{n-\frac{1}{2}}^{2}$;

for $i=4: Q_{n-\frac{1}{2}}^{1} \rightarrow\left(n^{2}+1 / 4\right) Q_{n-\frac{1}{2}}$ and

$P_{n-\frac{1}{2}}^{1} \rightarrow\left(n^{2}+1 / 4\right) P_{n-\frac{1}{2}}$

for $i=6: Q_{n-\frac{1}{2}}^{1} \rightarrow n Q_{n-\frac{1}{2}}^{1}$ and $P_{n-\frac{1}{2}}^{1} \rightarrow n P_{n-\frac{1}{2}}^{1}$;

for $i=5$ and $i=7$ it holds $a_{n}^{5}=a_{n}^{3}$ and $a_{n}^{7}=a_{n}^{6}$.

It follows from the last identities that

$G_{n}^{5}(\cosh \mu)=G_{n}^{3}(\cosh \mu)$ and $G_{n}^{7}(\cosh \mu)=G_{n}^{6}(\cosh \mu)$.

The coefficients $a_{0}^{i}$ can be arbitrary for $i=2,6$, and 7 .

\section{Appendix C: Diamagnetic force acting on a cylindrical flux rope}

Calculation of the diamagnetic force is straightforward. It follows from (A.10) that $B_{r}=0$ at the flux rope surface (for $r=r_{0}$ ). So (16) will be

$\boldsymbol{F}=-\frac{1}{2 \mu_{0}} \int_{-l / 2}^{l / 2} \int_{0}^{2 \pi}\left(B_{\varphi}^{2}+B_{Z}^{2}\right) \boldsymbol{n} r_{0} \mathrm{~d} \varphi \mathrm{d} Z$,

where $l$ is a flux rope length and the integral is calculated at $r=r_{0}$. The normal vector is expressed by using (5)-(7) and (A.1) as

$\boldsymbol{n}=(-\sin \varphi \sin \alpha, \sin \varphi \cos \alpha \cos \beta+\cos \varphi \sin \beta,-\cos \varphi \cos \beta+$ $\sin \varphi \cos \alpha \sin \beta)$.

The integration of (C.1) with (A.11)-(A.12) yields

$$
\begin{aligned}
F_{x}= & \frac{B_{0} B_{1} \pi r_{0}^{2} l}{\mu_{0} L} \sin ^{2} \alpha \\
& \times\left(2+\cos ^{2} \alpha-\cos ^{2} \beta+\cos ^{2} \alpha \cos ^{2} \beta\right), \\
F_{y}= & -\frac{B_{0} B_{1} \pi r_{0}^{2} l}{\mu_{0} L} \sin \alpha \cos \alpha \cos \beta \\
& \times\left(2+\cos ^{2} \alpha-\cos ^{2} \beta+\cos ^{2} \alpha \cos ^{2} \beta\right), \\
F_{z}= & -\frac{B_{0} B_{1} \pi r_{0}^{2} l}{\mu_{0} L} \sin \alpha \cos \alpha \sin \beta \\
& \times\left(2+\cos ^{2} \alpha-\cos ^{2} \beta+\cos ^{2} \alpha \cos ^{2} \beta\right) .
\end{aligned}
$$

The calculation of the moment of force (17) is also straightforward and gives

$$
\begin{aligned}
M_{x}= & -\frac{B_{1}^{2} \pi r_{0}^{2} l^{3}}{12 \mu_{0} L^{2}} \sin ^{2} \alpha \sin \beta \cos \beta \\
& \times\left(-2+\cos ^{2} \alpha-\cos ^{2} \beta+\cos ^{2} \alpha \cos ^{2} \beta\right), \\
M_{y}= & -\frac{B_{1}^{2} \pi r_{0}^{2} l^{3}}{12 \mu_{0} L^{2}} \sin \alpha \cos \alpha \sin \beta \\
& \times\left(2+\cos ^{2} \alpha-\cos ^{2} \beta+\cos ^{2} \alpha \cos ^{2} \beta\right), \\
M_{z}= & \frac{B_{1}^{2} \pi r_{0}^{2} l^{3}}{6 \mu_{0} L^{2}} \sin \alpha \cos \alpha \cos \beta \\
& \times\left(\cos ^{2} \alpha-\cos ^{2} \beta+\cos ^{2} \alpha \cos ^{2} \beta\right) .
\end{aligned}
$$

\section{Appendix D: Diamagnetic force acting on a toroidal flux rope}

The diamagnetic force acting on a toroid is given by (16) as

$\boldsymbol{F}=-\frac{1}{2 \tilde{\mu}_{0}} \oint\left(B_{\eta}^{2}+B_{\varphi}^{2}\right) \mathrm{d} \boldsymbol{S}$,

because it follows from (B.17) that $B_{\mu}=0$ at the flux rope surface (for $\mu=\mu_{0}$ ). We shall calculate the diamagnetic force (16) in the toroid's (tilde) system:

$\boldsymbol{F}=-\frac{1}{2 \tilde{\mu}_{0}} \int_{-\pi}^{\pi} \int_{0}^{2 \pi}\left(B_{\eta}^{2}+B_{\varphi}^{2}\right) \boldsymbol{w} \mathrm{d} \varphi \mathrm{d} \eta$

where

$w_{\tilde{x}}=\frac{a^{2} \sinh \mu_{0}\left(\cosh \mu_{0} \cos \eta-1\right)}{\left(\cosh \mu_{0}-\cos \eta\right)^{3}} \cos \varphi$,

$w_{\tilde{y}}=\frac{a^{2} \sinh \mu_{0}\left(\cosh \mu_{0} \cos \eta-1\right)}{\left(\cosh \mu_{0}-\cos \eta\right)^{3}} \sin \varphi$,

$w_{\tilde{z}}=\frac{a^{2} \sinh ^{2} \mu_{0} \sin \eta}{\left(\cosh \mu_{0}-\cos \eta\right)^{3}}$.

The integral in (D.2) and all subsequent integrals in this appendix are calculated at $\mu=\mu_{0}$.

The $\tilde{x}$ component is

$$
F_{\tilde{x}}=-\frac{1}{2 \tilde{\mu}_{0}} \int_{-\pi}^{\pi} \int_{0}^{2 \pi}\left(B_{\eta}^{2}+B_{\varphi}^{2}\right) w_{\tilde{x}} \mathrm{~d} \varphi \mathrm{d} \eta \equiv F_{\tilde{x} \eta}+F_{\tilde{x} \varphi}
$$

$$
\begin{aligned}
F_{\tilde{x} \eta}= & -\frac{a^{2} \sinh \mu_{0}}{2 \tilde{\mu}_{0}} \\
& \times \int_{-\pi}^{\pi} \int_{0}^{2 \pi} B_{\eta}^{2} \frac{\cosh \mu_{0} \cos \eta-1}{\left(\cosh \mu_{0}-\cos \eta\right)^{3}} \cos \varphi \mathrm{d} \varphi \mathrm{d} \eta, \\
F_{\tilde{x} \varphi}= & -\frac{a^{2} \sinh \mu_{0}}{2 \tilde{\mu}_{0}} \\
& \times \int_{-\pi}^{\pi} \int_{0}^{2 \pi} B_{\varphi}^{2} \frac{\cosh \mu_{0} \cos \eta-1}{\left(\cosh \mu_{0}-\cos \eta\right)^{3}} \cos \varphi \mathrm{d} \varphi \mathrm{d} \eta .
\end{aligned}
$$

Similar anotation will be used for the other components, i.e., $F_{\tilde{y}}=F_{\tilde{y} \eta}+F_{\tilde{y} \varphi}, F_{\tilde{z}}=F_{\tilde{z} \eta}+F_{\tilde{z} \varphi}$.

Substituting (B.19) into (D.7), we get

$$
\begin{aligned}
F_{\tilde{x} \varphi}= & -\frac{1}{2 \tilde{\mu}_{0} \sinh \mu_{0}} \sum_{i=1}^{7} \sum_{j=1}^{7} C_{i} C_{j} K_{i} K_{j} \\
& \times \int_{0}^{2 \pi} N_{i}^{\prime}(\varphi) N_{j}^{\prime}(\varphi) \cos \varphi \mathrm{d} \varphi \\
& \times \sum_{n=-\infty}^{\infty} \sum_{m=-\infty}^{\infty} G_{n}^{i}\left(\cosh \mu_{0}\right) G_{m}^{j}\left(\cosh \mu_{0}\right) \\
& \times \int_{-\pi}^{\pi}\left(\cosh \mu_{0} \cos \eta-1\right) H_{n}^{i}(\eta) H_{m}^{j}(\eta) \mathrm{d} \eta .
\end{aligned}
$$

The integrals over $\varphi$ in (D.8) are non-zero for the following pairs of $(i-j)$ : (1-3), (3-6), (5-7), and with swapped numbers; they are equal to $\pi$. For these combinations only the pair $(1-3)$ (and swapped (3-1)) gives a non-zero integral over $\eta$ in (D.8); 
for the others the integrals are zero due to odd functions in $\eta$. This one non-zero integral can be easily calculated over $\eta$ and the resulting expression is

$$
\begin{aligned}
F_{\tilde{x} \varphi}= & F_{\tilde{x} \varphi}^{(1-3)}=-\frac{4 B_{0} B_{1} a^{3}}{3 L \tilde{\mu}_{0} \sinh \mu_{0}} \\
& \times \sin \alpha\left(\cos ^{2} \alpha-\sin ^{2} \alpha \cos ^{2} \beta-2 \sin ^{2} \beta\right) \\
& \times \sum_{n=-\infty}^{\infty} G_{n}^{1}\left(\cosh \mu_{0}\right)\left\{\operatorname { c o s h } \mu _ { 0 } \left[G_{n-1}^{3}\left(\cosh \mu_{0}\right)\right.\right. \\
& \left.\left.+G_{n+1}^{3}\left(\cosh \mu_{0}\right)\right]-2 G_{n}^{3}\left(\cosh \mu_{0}\right)\right\} .
\end{aligned}
$$

Similar calculations yield the other components

$$
\begin{aligned}
F_{\tilde{y} \varphi}= & F_{\tilde{y} \varphi}^{(1-5)}=\frac{8 B_{0} B_{1} a^{3}}{3 L \tilde{\mu}_{0} \sinh \mu_{0}} \sin \alpha \cos \alpha \sin \beta \cos \beta \\
& \times \sum_{n=-\infty}^{\infty} G_{n}^{1}\left(\cosh \mu_{0}\right)\left\{\operatorname { c o s h } \mu _ { 0 } \left[G_{n-1}^{5}\left(\cosh \mu_{0}\right)\right.\right. \\
& \left.\left.+G_{n+1}^{5}\left(\cosh \mu_{0}\right)\right]-2 G_{n}^{5}\left(\cosh \mu_{0}\right)\right\}
\end{aligned}
$$

and

$$
\begin{aligned}
F_{\tilde{z} \varphi}= & F_{\tilde{z} \varphi}^{(1-6)}=\frac{16 B_{0} B_{1} a^{3}}{3 L \tilde{\mu}_{0}} \sin ^{2} \alpha \cos \alpha\left(1+\cos ^{2} \beta\right) \\
& \times \sum_{n=-\infty}^{\infty} G_{n}^{1}\left(\cosh \mu_{0}\right)\left[(n+1) G_{n+1}^{6}\left(\cosh \mu_{0}\right)\right. \\
& \left.-(n-1) G_{n-1}^{6}\left(\cosh \mu_{0}\right)\right] .
\end{aligned}
$$

Substituting (B.18) into (D.6) we get

$$
\begin{aligned}
F_{\tilde{x} \eta}= & -\frac{\sinh \mu_{0}}{8 \tilde{\mu}_{0}} \sum_{i=1}^{7} \sum_{j=1}^{7} C_{i} C_{j} K_{i} K_{j} \int_{0}^{2 \pi} N_{i}(\varphi) N_{j}(\varphi) \cos \varphi \mathrm{d} \varphi \\
& \times \sum_{n=-\infty}^{\infty} \sum_{m=-\infty}^{\infty} G_{n}^{i}\left(\cosh \mu_{0}\right) G_{m}^{j}\left(\cosh \mu_{0}\right) \\
& \times \int_{-\pi}^{\pi}\left[\sin \eta H_{n}^{i}(\eta)+2\left(\cosh \mu_{0}-\cos \eta\right) H_{n}^{i \prime}(\eta)\right] \\
& \times\left[\sin \eta H_{m}^{j}(\eta)+2\left(\cosh \mu_{0}-\cos \eta\right) H_{m}^{j \prime}(\eta)\right] \\
& \times \frac{\cosh \mu_{0} \cos \eta-1}{\left(\cosh \mu_{0}-\cos \eta\right)^{2}} \mathrm{~d} \eta .
\end{aligned}
$$

The integrals over $\varphi$ in (D.12) are non-zero for the following pairs of $(i-j)$ : (1-2), (1-4), (2-6), (4-6) (all equal to $\pi$ ), $(1-3),(3-6),(5-7)$ (all equal to $\pi / 2)$, and swapped. For these pairs only the integrals over $\eta$ in (D.6) for (1-3), (1-4), (2-6) (and swapped) are non-zero; the others are zero due to odd functions in $\eta$. The force (D.12) will be expressed as the sum $F_{\tilde{x} \eta}=F_{\tilde{x} \eta}^{(1-3)}+F_{\tilde{x} \eta}^{(1-4)}+F_{\tilde{x} \eta}^{(2-6)}$.

Calculations yield

$$
\begin{aligned}
F_{\tilde{x} \eta}^{(i-j)}= & f_{\tilde{x} \eta}^{(i-j)} \sum_{m=-\infty}^{\infty} I_{m} \sum_{n=-\infty}^{\infty}\left[p_{n}^{i}\left(p_{n-m}^{j} \mp p_{m-n-2}^{j}-r_{n-m+1}^{j}\right)\right. \\
& \mp p_{-n}^{i}\left(p_{n-m-2}^{j} \mp p_{m-n}^{j}-r_{n-m-1}^{j}\right) \\
& \left.-r_{n}^{i}\left(p_{n-m-1}^{j} \mp p_{m-n-1}^{j}-r_{n-m}^{j}\right)\right],
\end{aligned}
$$

where the upper signs hold for the pairs (1-3) and (1-4), and the lower signs for the pair (2-6). Coefficients $p_{n}^{i}$ and $r_{n}^{i}$ are defined

for $i=1$ and 3

$p_{n}^{i}=(2 n+1) G_{n}^{i}\left(\cosh \mu_{0}\right)$,

$r_{n}^{i}=4 n \cosh \mu_{0} G_{n}^{i}\left(\cosh \mu_{0}\right)$,

for $i=2$ and 6

$p_{n}^{i}=(2 n+1) n G_{n}^{i}\left(\cosh \mu_{0}\right)$,

$r_{n}^{i}=4 n^{2} \cosh \mu_{0} G_{n}^{i}\left(\cosh \mu_{0}\right)$,

and for $i=4$

$p_{n}^{4}=(2 n+1)\left(n^{2}+\frac{1}{4}\right) G_{n}^{4}\left(\cosh \mu_{0}\right)$,

$r_{n}^{4}=4 n\left(n^{2}+\frac{1}{4}\right) \cosh \mu_{0} G_{n}^{4}\left(\cosh \mu_{0}\right)$.

The remaining symbols are equal to

$f_{\tilde{x} \eta}^{(1-3)}=-\frac{B_{0} B_{1} a^{3} \sinh \mu_{0}}{24 \pi L \tilde{\mu}_{0}}$ $\times \sin \alpha\left(\cos ^{2} \alpha-\sin ^{2} \alpha \cos ^{2} \beta-2 \sin ^{2} \beta\right)$,

$f_{\tilde{x} \eta}^{(1-4)}=-\frac{B_{0} B_{1} a^{3} \sinh \mu_{0}}{4 \pi L \tilde{\mu}_{0}} \sin \alpha\left(\cos ^{2} \alpha-\sin ^{2} \alpha \cos ^{2} \beta\right)$,

$f_{\tilde{x} \eta}^{(2-6)}=\frac{B_{0} B_{1} a^{3} \sinh \mu_{0}}{3 \pi L \tilde{\mu}_{0}} \sin \alpha \cos ^{2} \alpha\left(1+\cos ^{2} \beta\right)$,

and

$I_{n}=\int_{-\pi}^{\pi} \frac{\cosh \mu_{0} \cos \eta-1}{\left(\cosh \mu_{0}-\cos \eta\right)^{2}} \cos n \eta \mathrm{d} \eta$

$=2 \pi|n|\left(\cosh \mu_{0}-\sinh \mu_{0}\right)^{|n|}$.

During the derivations relationships like these were used:

$p_{-n}^{3}=-(2 n-1) G_{n}^{3}\left(\cosh \mu_{0}\right), r_{-n}^{3}=-r_{n}^{3}$, etc.

The other force components are calculated similarly. We have

$F_{\tilde{y} \eta}=F_{\tilde{y} \eta}^{(1-5)}+F_{\tilde{y} \eta}^{(2-7)}$

$F_{\tilde{z} \eta}=F_{\tilde{z} \eta}^{(1-6)}+F_{\tilde{z} \eta}^{(4-2)}$.

For $F_{\tilde{y} \eta}^{(i-j)}$ the same expression holds as (D.14), where the upper signs are for the pair (1-5) and the lower signs for the pair $(2-7), p_{n}^{5}=p_{n}^{3} r_{n}^{5}=r_{n}^{3}, p_{n}^{7}=p_{n}^{6} r_{n}^{7}=r_{n}^{6}$, and

$f_{\tilde{y} \eta}^{(1-5)}=\frac{B_{0} B_{1} a^{3} \sinh \mu_{0}}{12 \pi L \tilde{\mu}_{0}} \sin \alpha \cos \alpha \sin \beta \cos \beta$,

$f_{\tilde{y} \eta}^{(2-7)}=-\frac{B_{0} B_{1} a^{3} \sinh \mu_{0}}{3 \pi L \tilde{\mu}_{0}} \sin \alpha \cos \alpha \sin \beta \cos \beta$.

The parts of the $F_{\tilde{z} \eta}$ component are equal to

$$
\begin{aligned}
F_{\tilde{z} \eta}^{(i-j)}= & f_{\tilde{z} \eta}^{(i-j)} \sum_{m=-\infty}^{\infty} J_{m} \sum_{n=-\infty}^{\infty}\left[2 s_{n}^{i}\left(p_{n-m-1}^{j}+p_{m-n-1}^{j}-r_{n-m}^{j}\right)\right. \\
& -p_{n}^{i}\left(p_{n-m+1}^{j}+p_{m-n-3}^{j}-r_{n-m+2}^{j}\right) \\
& -p_{-n}^{i}\left(p_{n-m-3}^{j}+p_{m-n+1}^{j}-r_{n-m-2}^{j}\right)-r_{n}^{i}\left(p_{n-m-2}^{j}\right. \\
& \left.-p_{m-n-2}^{j}-p_{n-m}^{j}+p_{m-n}^{j}+r_{n-m+1}^{j}-r_{n-m-1}^{j}\right),
\end{aligned}
$$


where $s_{n}^{i}$ are defined

for $i=1,3$, and 5

$s_{n}^{i}=G_{n}^{i}\left(\cosh \mu_{0}\right)$,

for $i=4$

$s_{n}^{4}=\left(n^{2}+\frac{1}{4}\right) G_{n}^{4}\left(\cosh \mu_{0}\right)$,

and for $i=6$ and 7

$s_{n}^{i}=n G_{n}^{i}\left(\cosh \mu_{0}\right)$.

The remaining symbols are equal to

$f_{\tilde{z} \eta}^{(1-6)}=-\frac{B_{0} B_{1} a^{3} \sinh ^{2} \mu_{0}}{6 \pi L \tilde{\mu}_{0}} \sin ^{2} \alpha \cos \alpha\left(1+\cos ^{2} \beta\right)$,

$f_{\tilde{z} \eta}^{(4-2)}=\frac{B_{0} B_{1} a^{3} \sinh ^{2} \mu_{0}}{4 \pi L \tilde{\mu}_{0}} \cos \alpha\left(\cos ^{2} \alpha-\sin ^{2} \alpha \cos ^{2} \beta\right)$,

and

$$
\begin{aligned}
J_{n} & =\int_{-\pi}^{\pi} \frac{\cos n \eta}{\left(\cosh \mu_{0}-\cos \eta\right)^{2}} \mathrm{~d} \eta \\
& =\frac{2 \pi}{\sinh ^{3} \mu_{0}}\left(\cosh \mu_{0}-\sinh \mu_{0}\right)^{|n|}\left(\cosh \mu_{0}+|n| \sinh \mu_{0}\right) .
\end{aligned}
$$

The integral $I_{n}$ (D.15) can be expressed through $J_{n}$ by

$I_{n}=\frac{1}{2} \cosh \mu_{0}\left(J_{n+1}+J_{n-1}\right)-J_{n}$.

The force in the original system $x y z$ can be obtained by applying the transformations (5)-(7). Due to symmetry $F_{z}=0$.

The moment of diamagnetic force (17),

$\boldsymbol{M}=-\frac{1}{2 \tilde{\mu}_{0}} \oint\left(B_{\eta}^{2}+B_{\varphi}^{2}\right)(\boldsymbol{r} \times \mathrm{d} \boldsymbol{S})$

will be also calculated in the toroid's (tilde) system:

$M_{\tilde{x}}=-\frac{1}{2 \tilde{\mu}_{0}} \int_{-\pi}^{\pi} \int_{0}^{2 \pi}\left(B_{\eta}^{2}+B_{\varphi}^{2}\right)\left(\tilde{y} w_{\tilde{z}}-\tilde{z} w_{\tilde{y}}\right) \mathrm{d} \varphi \mathrm{d} \eta$,

$M_{\tilde{y}}=-\frac{1}{2 \tilde{\mu}_{0}} \int_{-\pi}^{\pi} \int_{0}^{2 \pi}\left(B_{\eta}^{2}+B_{\varphi}^{2}\right)\left(\tilde{z} w_{\tilde{x}}-\tilde{x} w_{\tilde{z}}\right) \mathrm{d} \varphi \mathrm{d} \eta$,

$M_{\tilde{z}}=-\frac{1}{2 \tilde{\mu}_{0}} \int_{-\pi}^{\pi} \int_{0}^{2 \pi}\left(B_{\eta}^{2}+B_{\varphi}^{2}\right)\left(\tilde{x} w_{\tilde{y}}-\tilde{y} w_{\tilde{x}}\right) \mathrm{d} \varphi \mathrm{d} \eta$.

A direct substitution of (B.1)-(B.3) and (D.3)-(D.5) into (D.24) gives immediately $M_{\tilde{z}}=0$. For the remaining moment of force components we shall proceed similarly to the calculation of the diamagnetic force:

$M_{\tilde{x}}=M_{\tilde{x} \varphi}+M_{\tilde{x} \eta}$,

$M_{\tilde{x} \varphi}=M_{\tilde{x} \varphi}^{(3-7)}+M_{\tilde{x} \varphi}^{(5-6)}$,

$M_{\tilde{x} \eta}=M_{\tilde{x} \eta}^{(3-7)}+M_{\tilde{x} \eta}^{(4-7)}+M_{\tilde{x} \eta}^{(5-6)}$,

$M_{\tilde{y}}=M_{\tilde{y} \varphi}+M_{\tilde{y} \eta}$,

$M_{\tilde{y} \varphi}=M_{\tilde{y} \varphi}^{(3-6)}+M_{\tilde{y} \varphi}^{(5-7)}$,
$M_{\tilde{y} \eta}=M_{\tilde{y} \eta}^{(1-2)}+M_{\tilde{y} \eta}^{(3-6)}+M_{\tilde{y} \eta}^{(4-6)}+M_{\tilde{y} \eta}^{(5-7)}$

the other $(i-j)$ pairs give zero contribution because their integrals over $\varphi$ or $\eta$ are zero;

$M_{\tilde{x} \varphi}^{(i-j)}=A_{\tilde{x} \varphi}^{(i-j)} \sum_{n=-\infty}^{\infty} G_{n}^{i}\left(\cosh \mu_{0}\right)\left(s_{n+1}^{j}-s_{n-1}^{j}\right)$

the expression for $M_{\tilde{x} \eta}^{(i-j)}$ has exactly the same form as (D.18) with $F_{\tilde{z} \eta}^{(i-j)}$ and $f_{\tilde{z} \eta}^{(i-j)}$ replaced by $M_{\tilde{x} \eta}^{(i-j)}$ and $A_{\tilde{x} \eta}^{(i-j)}$, respectively. The same expressions as for $M_{\tilde{x} \varphi}^{(i-j)}$ and $M_{\tilde{x} \eta}^{(i-j)}$ hold for the $\tilde{y}$ components. Constants $A^{(i-j)}$ are equal to

$A_{\tilde{x} \varphi}^{(3-7)}=\frac{8 B_{1}^{2} a^{5} \cosh \mu_{0}}{9 L^{2} \tilde{\mu}_{0} \sinh \mu_{0}} \sin \alpha \sin \beta \cos \beta$

$\times\left(\cos ^{2} \alpha-\sin ^{2} \alpha \cos ^{2} \beta-2 \sin ^{2} \beta\right)$,

$A_{\tilde{x} \varphi}^{(5-6)}=-\frac{16 B_{1}^{2} a^{5} \cosh \mu_{0}}{9 L^{2} \tilde{\mu}_{0} \sinh \mu_{0}} \sin \alpha \cos ^{2} \alpha \sin \beta \cos \beta\left(1+\cos ^{2} \beta\right)$,

$A_{\tilde{y} \varphi}^{(3-6)}=-\frac{8 B_{1}^{2} a^{5} \cosh \mu_{0}}{9 L^{2} \tilde{\mu}_{0} \sinh \mu_{0}} \sin \alpha \cos \alpha$

$\times\left(\cos ^{2} \alpha-\sin ^{2} \alpha \cos ^{2} \beta-2 \sin ^{2} \beta\right)\left(1+\cos ^{2} \beta\right)$,

$A_{\tilde{y} \varphi}^{(5-7)}=-\frac{16 B_{1}^{2} a^{5} \cosh \mu_{0}}{9 L^{2} \tilde{\mu}_{0} \sinh \mu_{0}} \sin \alpha \cos \alpha \sin ^{2} \beta \cos ^{2} \beta$,

$A_{\tilde{x} \eta}^{(3-7)}=-\frac{B_{1}^{2} a^{5} \sinh \mu_{0} \cosh \mu_{0}}{72 \pi L^{2} \tilde{\mu}_{0}} \sin \alpha \sin \beta \cos \beta$

$\times\left(\cos ^{2} \alpha-\sin ^{2} \alpha \cos ^{2} \beta-2 \sin ^{2} \beta\right)$,

$A_{\tilde{x} \eta}^{(4-7)}=\frac{B_{1}^{2} a^{5} \sinh \mu_{0} \cosh \mu_{0}}{12 \pi L^{2} \tilde{\mu}_{0}} \sin \alpha \sin \beta \cos \beta$

$\times\left(\cos ^{2} \alpha-\sin ^{2} \alpha \cos ^{2} \beta\right)$,

$A_{\tilde{x} \eta}^{(5-6)}=\frac{B_{1}^{2} a^{5} \sinh \mu_{0} \cosh \mu_{0}}{36 \pi L^{2} \tilde{\mu}_{0}} \sin \alpha \cos ^{2} \alpha \sin \beta \cos \beta$

$\times\left(1+\cos ^{2} \beta\right)$,

$A_{\tilde{y} \eta}^{(1-2)}=-\frac{B_{0}^{2} a^{3} \sinh \mu_{0} \cosh \mu_{0}}{4 \pi \tilde{\mu}_{0}} \sin \alpha \cos \alpha$,

$A_{\tilde{y} \eta}^{(3-6)}=\frac{B_{1}^{2} a^{5} \sinh \mu_{0} \cosh \mu_{0}}{72 \pi L^{2} \tilde{\mu}_{0}} \sin \alpha \cos \alpha$

$\times\left(\cos ^{2} \alpha-\sin ^{2} \alpha \cos ^{2} \beta-2 \sin ^{2} \beta\right)\left(1+\cos ^{2} \beta\right)$,

$A_{\tilde{y} \eta}^{(4-6)}=\frac{B_{1}^{2} a^{5} \sinh \mu_{0} \cosh \mu_{0}}{12 \pi L^{2} \tilde{\mu}_{0}} \sin \alpha \cos \alpha$

$\times\left(\cos ^{2} \alpha-\sin ^{2} \alpha \cos ^{2} \beta\right)\left(1+\cos ^{2} \beta\right)$,

$A_{\tilde{y} \eta}^{(5-7)}=\frac{B_{1}^{2} a^{5} \sinh \mu_{0} \cosh \mu_{0}}{36 \pi L^{2} \tilde{\mu}_{0}} \sin \alpha \cos \alpha \sin ^{2} \beta \cos ^{2} \beta$. 


\section{References}

Altschuler, M. D., \& Newkirk, G. 1969, Sol. Phys., 9, 131 Bagenal, F., \& Gibson, S. 1991, J. Geophys. Res., 96, 17663 Chen, J., Howard, R. A., Brueckner, G. E., et al. 1997, ApJ, 490, L191 Hoeksema, J. T. 1984, Tech. Rep. CSSA-ASTRO-84-07 (California: Stanford Univ.)

Klein, L. W., \& Burlaga, L. F. 1982, J. Geophys. Res., 87, 613

Klimchuk, J. A., Antiochos, S. K., \& Norton, D. 2000, ApJ, 542, 504

Low, B. C., \& Hundhausen, J. R. 1995, ApJ, 443, 818

Marubashi, K. 1986, Adv. Space Res., 6(6), 335

Parker, E. N. 1957, ApJS, 3, 51

Pneuman, G. W. 1983, ApJ, 265, 468
Romashets, E. P., \& Vandas, M. 2001, J. Geophys. Res., 106, 10615

Romashets, E. P., \& Vandas, M. 2003, in Solar Wind Ten, ed. M. Velli, R. Bruno, \& F. Malara, AIP Conf. Proc., 679, 180

Sarris, E. T., \& Krimigis, S. 1982, Geophys. Res. Lett., 9, 167

Shatten, K. H. 1971, Cosmic Electrodynamics, 2, 232

Shimazu, H., \& Marubashi, K. 2000, J. Geophys. Res., 105, 2365

Vandas, M., Watari, S., \& Geranios, A. 2003, in Solar Wind Ten, ed. M. Velli, R. Bruno, \& F. Malara, AIP Conf. Proc., 679, 691

Vršnak, B., Ruždjak, V., Brajša, R., \& Džubur, A. 1988, Sol. Phys., 116,45

Zhao, X., \& Hoeksema, J. T. 1992, Sol. Phys., 143, 41

Zhao, X., \& Hoeksema, J. T. 1994, Sol. Phys., 151, 91 